\title{
THE BEST-ANNOTATION AWARD 1993
}

\author{
The Board of the ICCA
}

The Board of the ICCA is pleased to have received two entries for the first Best-Annotation Award, announced in the ICCA Journal, Vol. 15, No. 4, pp. 235-236 and reproduced in the ICCA Journal, Vol. 17, No. 1, pp. 39-40. These entries were THE CHESSMASTER 4000 TURBo (by The Software Toolworks, Inc., Burgess Hill, West Sussex, UK) and M-Chess Pro 3.5 (by M. Hirsch, San Rafael, CA, USA). The game set to be annotated was the $10^{\text {th }}$ game of the PCA match between Kasparov and Short. The process of adjudication has been conducted as follows. Entrants have submitted their programs on diskettes to David Levy. It was the latter who determined the game to be annotated. Needless to say, entrants were unaware which game this was when they submitted their programs. Subsequently, the program moves were entered manually by the Deputy Editor of this Journal. Under his supervision, the programs entered were run against the moves so recorded. With the independence of the game selected and the programs entered thus guaranteed, the output for an evaluation by the jury was produced. While M-CHESS PRO 3.5 produced a printout of the principal continuations and scores after each move, it was only THE CHESSMASTER 4000 TURBO which provided genuine annotations as chess-players understand the term.

Accordingly, the jury, nominated by the Board and consisting of David Levy, Jonathan Schaeffer and Jaap van den Herik, declares THE CHESSMASTER 4000 TURBO to be the first winner of the Best-Annotation Award. Below, we reproduce the annotations provided by THE CHESSMASTER 4000 TURBO, running on a 66 $\mathrm{MHz} 486 \mathrm{DX} 2$, at 120 seconds per move.

Owing to difficulties in the definition of our sponsorship in the Journal, the material award of the winning program must be limited to US\$ $100 .-$, sponsored anonymously. The ICCA is currently contemplating a project in which the winning program would provide an automatic annotation of a match yet to be played. Such a match must be of interest to the computer-chess community at large and might well be a match between the reigning human champion and his computer counterpart. If credibly produced, the ICCA undertakes to promote the commercial publication of the annotation, all benefits to accrue to the program's author.

The winner's output follows in the ICCA Journal's typesetting.

1. $\mathrm{e} 4 \quad \ldots$

... $\quad c 5$

2. Nf3 ... .. d6

3. $\mathrm{d} 4 \mathrm{~N}$

4. $\quad \cdots \quad c x d 4$

4. $\mathrm{Nxd} 4 \quad \ldots$

... Nf6

5. $\mathrm{Nc3} \quad \ldots$

6. $\quad$ a6

6. $\mathrm{Bc} 4 \quad \cdots$

7. $\quad \mathrm{Bb} 3 \quad \mathrm{e} 6$

N8d7

The King's Pawn opening move is both popular and logical. It controls the center, opens lines for both the Queen and the Bishop, and usually leads to an open game in which tactics, rather than maneuvering, predominates.

The Sicilian Defense has an ancient lineage. It creates an unbalanced position in which both sides have full rein for play, and allows Black to call the shots at least to some extent.

White's normal response prepares $\mathrm{d} 4$ and avoids such committing moves as $\mathrm{f} 4 \mathrm{fr}$ d3 or Nc3, which have their own rationale.

The Dragon Variation establishes a "dragon-like" pawn chain including g6, fianchettoing the King's Bishop. The d6 pawn is necessary to prevent e5. Sicilian Defense / Dragon Variation

Sicilian Defense / Dragon Variation

Sicilian Defense / Dragon Variation

The Knight attack on the eA pawn forces White to commit to its protection.

Sicilian Defense / Dragon Variation

Missing Annotation

Missing Annotation

Sicilian Defense / Najdorf Variation / 6 Bc4

Sicilian Defense / Najdorf Variation / 6 Bc4

Out of Opening Book. 


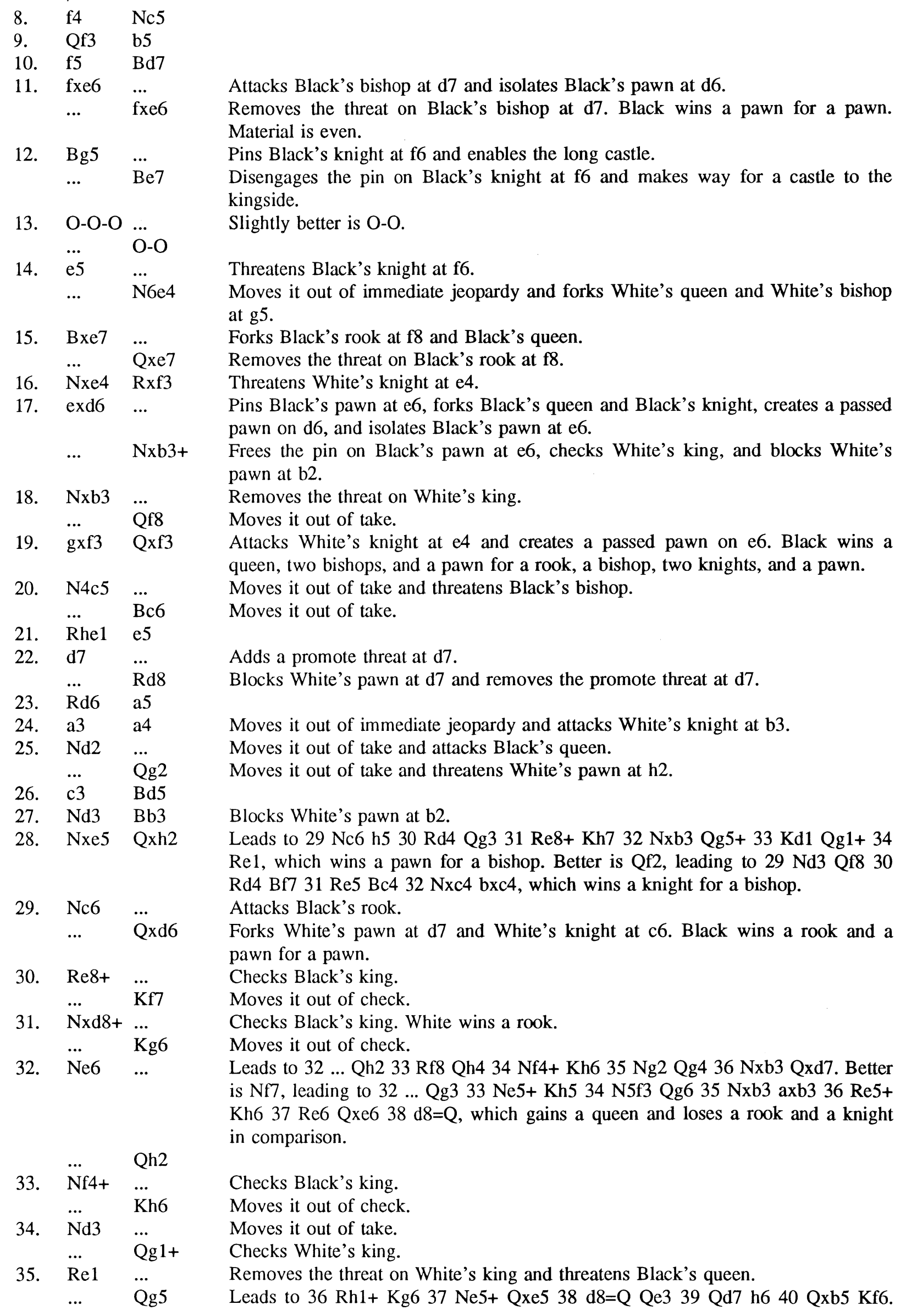


36. Ne5 $\quad$...

\begin{tabular}{|c|c|c|}
\hline \multirow{3}{*}{37.} & & g6 \\
\hline & Rf1 & \\
\hline & $\ldots$ & Be6 \\
\hline \multirow[t]{2}{*}{38.} & Nf7+ & \\
\hline & $\ldots$ & $\mathrm{Bxf} 7$ \\
\hline \multirow[t]{2}{*}{39.} & Rxf7 & \\
\hline & $\ldots$ & Qd5 \\
\hline \multirow[t]{2}{*}{40.} & $\operatorname{Re} 7$ & $\ldots$ \\
\hline & $\ldots$ & Qd6 \\
\hline \multirow[t]{2}{*}{41.} & Rf7 & $\ldots$ \\
\hline & $\ldots$ & Qd3 \\
\hline & $\mathrm{Ne} 4$ & Qe3+ \\
\hline \multirow[t]{2}{*}{43.} & $\mathrm{Nd} 2$ & \\
\hline & 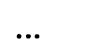 & Qd3 \\
\hline
\end{tabular}

Better is Qb6, leading to $36 \mathrm{Nxb3}$ Qd8 $37 \mathrm{Ne} 5 \mathrm{Qh} 438 \mathrm{Nf7}+\mathrm{Kg} 639 \mathrm{Rf} 1 \mathrm{Qh} 3$, which gains a queen and a pawn and loses a bishop, a knight, and a pawn in comparison.

Leads to 36 ... g6 37 d8=Q Qxd8 38 Nxb3 Qg5+ 39 Nd2 Qf5 $40 \mathrm{Kd1}$ Kg7 41 N2f3. Better is Rh1+, leading to 36 ... Kg6 $37 \mathrm{Ne} 5+$ Qxe5 38 d8=Q Qe3 39 Qd7 h6 40 Qxb5 Kf6, which gains a queen and a pawn and loses a bishop and a knight in comparison.

Takes control of the open file.

Slightly better is Rf8.

Removes the threat on Black's king and Black's queen.

White wins a bishop for a knight.

Attacks White's rook.

Moves it out of take and seizes the open file.

Threatens White's rook.

Moves it out of take and seizes the open file.

Checks White's king.

Moves it out of harm's way and removes the threat on White's king.

We note that THE CHESSMASTER 4000 TURBO recommends 36. Rh1+ which was the move suggested by Short after the game (see the Diagram below).

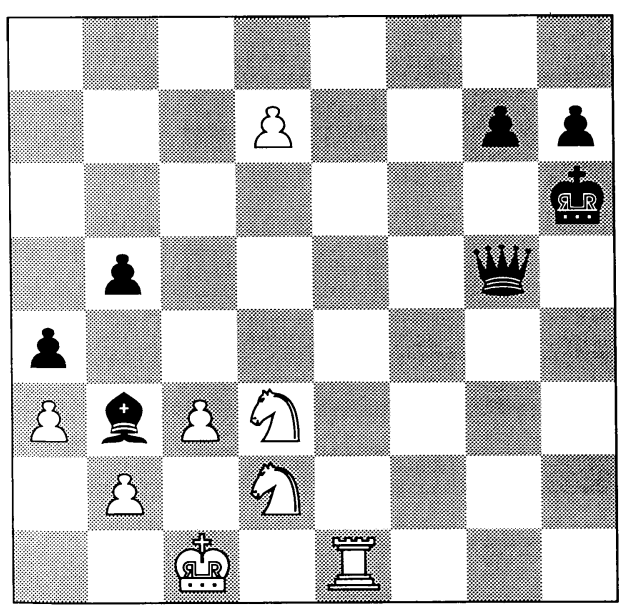

Short - Kasparov $\left(10^{\text {th }}\right.$ game $)$

Position after 35. ... Qg5.

The jury comments: "We feel that, although the annotations by the winning entry are somewhat primitive, they represent an important first step in the art of computerized annotation. With the strength of commercially available chess programs continuing to rise steadily, it is clear that one of the most important uses of such programs in the future will be the annotation of the users' games and instruction to help the users improve their playing ability. It cannot be many years before the winner of the ICCA Best-Annotation Award is able to tell the users to place more emphasis on the pawn structure around their King or to avoid castling on the flank where their opponent already has the makings of an attack against that King." 